九州大学学術情報リポジトリ

Kyushu University Institutional Repository

Association of MTH1 expression with the tumor malignant potential and poor prognosis in patients with resected lung cancer

藤下，卓才

ht tps://doi. org/10.15017/1928628

出版情報：九州大学，2017，博士（医学），課程博士

バージョン：

権利関係 : ๑ 2017 Elsevier B.V. All rights reserved. 


\title{
Association of MTH1 expression with the tumor malignant potential and poor prognosis in patients with resected lung cancer
}

\author{
Takatoshi Fujishita ${ }^{\mathrm{a}}$, Tatsuro Okamoto ${ }^{\mathrm{a}, *}$, Takaki Akamine $^{\mathrm{a}}$, Shinkichi Takamori ${ }^{\mathrm{a}}$, \\ Kazuki Takada ${ }^{\mathrm{a}, \mathrm{c}}$, Masakazu Katsura ${ }^{\mathrm{a}}$, Goji Toyokawa ${ }^{\mathrm{a}}$, Fumihiro Shoji ${ }^{\mathrm{a}}$, Mototsugu Shimokawa ${ }^{\mathrm{d}}$, \\ Yoshinao Oda ${ }^{\mathrm{c}}$, Yusaku Nakabeppu ${ }^{\mathrm{b}}$, Yoshihiko Maehara ${ }^{\mathrm{a}}$ \\ ${ }^{a}$ Department of Surgery and Science, Graduate School of Medical Sciences, Kyushu University, Fukuoka, Japan \\ b Division of Neurofunctional Genomics, Department of Immunobiology and Neuroscience, Medical Institute of Bioregulation, Kyushu University, Fukuoka, Japan \\ c Department of Anatomic Pathology, Graduate School of Medical Sciences, Kyushu University, Fukuoka, Japan \\ d Clinical Research Institute, National Kyushu Cancer Center, Fukuoka, Japan
}

\section{A R T I C L E I N F O}

\section{Keywords:}

MutT Homolog 1 (MTH1)

Non-small cell lung cancer (NSCLC)

Oxidative stress

Surgery

\begin{abstract}
A B S T R A C T
Objectives: The oxidized purine nucleoside triphosphatase, mutT homolog 1 (MTH1), physiologically sanitizes 8 oxo-dGTP in the nucleotide pool. Previous studies indicated that MTH1 is associated with tumor proliferation and invasion in non-small cell lung cancer (NSCLC) cell lines; however, the role of MTH1 in patients with NSCLC remains unclear.

Materials and Methods: Two patient cohorts that underwent surgery for NSCLC in our institution were investigated retrospectively. In one cohort consisting of 197 patients, the associations between MTH1 expression and clinicopathological factors or prognosis were analyzed. In another cohort consisting of 41 patients, the relationship between MTH1 expression in the tumors and serum oxidative stress levels (evaluated by the diacron-reactive oxygen metabolites [d-ROMs] test) or antioxidant capacity in the patients (evaluated by the biological antioxidant potential (BAP) test) was analyzed. A total of 238 patients were assessed for MTH1 protein levels using immunohistochemistry.

Results: Among the 197 patients in the former cohort, 111 (56.3\%) exhibited high MTH1 expression, while 86 (43.7\%) exhibited low MTH1 expression. Male sex, smoking habit of $\geq 20$ pack-years, squamous cell carcinoma, pathological stage $\geq$ II, tumor diameter $\geq 30 \mathrm{~mm}$, lymph node metastases, pleural invasion, lymphatic permeation and vascular infiltration were significantly associated with high MTH1 expression $(p<0.05)$. The high MTH1 expression group had a significantly worse prognosis than that of the low MTH1 expression group (5-year overall survival: $81.6 \%$ vs. $92.3 \%, p=0.0011$; 5-year disease-free survival: $55.0 \%$ vs. $83.7 \%$, $p=0.0002)$. d-ROMs and BAP test values were significantly higher in the high than in the low MTH1 expression group $(p<0.05)$.

Conclusion: This study showed that MTH1 protein expression was closely related to factors associated with a high malignant potential and poor patient survival. MTH1 may be a novel therapeutic target for NSCLC.
\end{abstract}

\section{Introduction}

Lung cancer is the leading cause of cancer-related death worldwide [1]. Importantly, it has been found that some subsets of lung cancer, especially adenocarcinoma, are driven by oncogenes, such as epidermal growth factor receptor (EGFR), Kirsten rat sarcoma viral oncogene homolog (KRAS) and anaplastic lymphoma kinase (ALK) [2-4]. Based on these discoveries, molecular targeted drugs for lung cancer, including EGFR-tyrosine kinase inhibitors (TKIs) and ALK-TKIs, have been developed and applied clinically [4-6]. Despite these successful ad- vances in the field of molecular oncology, the concise mechanisms by which mutations develop in carcinogenesis have yet to be elucidated. Recently, several cellular environmental or intrinsic stresses, including oxidative stress, hypoxia, and epigenetic disorders, have received much attention as potential contributors to lung carcinogenesis $[7,8]$.

Reactive oxygen species (ROS), produced during oxygen respiration and other normal metabolic processes or inflammatory responses, oxidize nucleotides and proteins, thus inducing genetic mutations and cellular senescence $[9,10]$. With respect to endogenous oxidation of DNA and DNA precursor nucleotides, ROS produce 8-oxo-guanine (8-

\footnotetext{
* Corresponding author at: Department of Surgery and Science, Graduate School of Medical Sciences Kyushu University 3-1-1, Maidashi, Higashi-ku, Fukuoka 812-8582, Japan.

E-mail address: tatsuro@surg2.med.kyushu-u.ac.jp (T. Okamoto).
} 
oxoG) by oxidizing dGTP in the nucleotide pool or directly oxidizing guanine bases in DNA. DNA polymerases often insert 8-oxo-dGTP opposite adenine as well as cytosine in template DNA during DNA replication, thus leading to $\mathrm{A}$ to $\mathrm{C}$ or $\mathrm{G}$ to $\mathrm{T}$ transversion mutations [11]. Accumulation of these DNA mutations can induce carcinogenesis or cellular dysfunction. To avoid such deleterious outcomes, human cells are equipped with several molecules that sanitize the oxidized nucleotides. MutT homolog 1 (MTH1), an oxidized purine nucleoside triphosphatase, hydrolyzes 8-oxo-dGTP to its monophosphate form, which is no longer utilized by DNA polymerases, thus preventing its genomic incorporation [11-13]. MTH1-deficient mice exhibited increased susceptibility to spontaneous tumorigenesis in the lung, liver and stomach compared with wild-type mice [14]. This finding suggests a potential role of MTH1 as a tumor suppressor.

Some specific cellular environments, such as exposure to tobacco smoke or ionizing radiation, increase ROS levels in normal cells $[8,9]$. In addition, ROS are also generated as by-products of metabolic processes of the cellular respiratory chain or as molecular executors of host defenses. Since cancer cells proliferate rapidly, they tend to reorganize cellular metabolic pathways as they grow. As a consequence, cancer cells are always exposed to high ROS levels [15], which can lead to 8-oxo-GTP accumulation in the nucleotide pool. Since excessive accumulation of 8-oxoG in DNA can induce cell death $[11,16]$, cancer cells may acquire mechanisms to prevent the accumulation of 8-oxoG in DNA and thereby oxidative stress. In fact, MTH1 is abundant in various types of cancers, such as renal cell, lung, brain, breast and gastric cancers [17-21]. Moreover, according to a preclinical study, MTH1 was required for the proliferation of cancer cells [22]. Another study recently demonstrated that MTH1 plays a pro-malignancy role in KRAS-driven non-small-cell lung cancer (NSCLC) cells [23]. In xenograft models, targeting MTH1 inhibited the proliferation of tumors that developed from tumor cell lines [22,24].

These findings suggest that MTH1 plays an important role in cancer development; however, very few data have demonstrated the clinical significance of MTH1 expression in primary lung cancer so far. In this study, we examined MTH1 protein expression in 197 resected NSCLC cases by immunohistochemistry and the potential association between MTH1 expression and clinicopathological factors, survival, and oxidative stress in patients.

\section{Materials and methods}

\subsection{Patients}

Two cohorts of NSCLC patients were enrolled in this retrospective study. One cohort (Cohort A) included 197 patients who underwent surgical resection but did not receive any preoperative induction treatment between March 2003 and December 2006 in the Department of Surgery and Science, Kyushu University Hospital. Another cohort (Cohort B) consisted of 41 patients who underwent surgical resection for NSCLC and underwent tests for diacron-reactive oxygen metabolites (d-ROMs) and biological antioxidant potential (BAP) between May 2008 and April 2010 [25]. The seventh edition of the TNM Classification was used for pathological staging. Various clinicopathological factors were extracted from medical records, such as age, sex, smoking history, body mass index, adjuvant therapy, pathological diagnosis, interstitial pneumonia, and EGFR mutation status. After surgery, routine check-ups, including a physical examination, blood tests (including serum tumor markers), and chest x-ray, were performed at 3-month intervals for the first 3 years and at 6month intervals thereafter. Computed tomography was performed twice a year for the first 3 years and at least annually thereafter. Clinical information and follow-up data were obtained from medical records. Forty- five (22.8\%) patients in Cohort A underwent fludeoxyglucose positron emission tomography (FDG-PET/CT) before surgery.

The institutional review board of our institution approved this study (Kyushu-university IRB \#28-253).

\subsection{Immunohistochemistry}

A specific rabbit antibody raised against recombinant human MTH1 (anti-MTH1) [19,26,27] was used for immunohistochemical analysis. After deparaffinization, specimens were treated with peroxidase blocking reagent and protein blocking reagent (080-01186 Wako, Osaka, Japan and 19-2410-3 Sigma-Aldrich, Tokyo, Japan, respectively). Thereafter, tissue sections were incubated with the anti-MTH1 antibody at a dilution of $1: 100$, followed by DAKO ENVISION + polymer/HRP (anti-rabbit; K4003 Dako, Tokyo, Japan). Liquid DAB + chromogen (049-22831, Wako, Osaka, Japan) was used for visualization of the antigen. Finally, tissue specimens were stained with hematoxylin (30002, Muto Pure Chemicals, Tokyo, Japan) to distinguish the nucleus from the cytoplasm.

The proportion of cells exhibiting MTH1 staining among the overall cancer cells was determined and scored as follows: $1,0-19 \% ; 2$, $20-39 \%$; 3, 40-59\%; 4, 60-79\%; 5, 80-100\%. Receiver operating characteristic (ROC) curve analysis was performed to predict overall survival (OS) and disease-free survival (DFS) according to the MTH1 staining score. Score 3 exhibited the closest association with both the maximum sensitivity and specificity and thus was selected as the cut-off score for MTH1 expression (Supplementary Figure 1). According to the ROC curves, cases were judged to be positive or negative for MTH1 expression when $\geq$ or $<60 \%$ of the tumor cells were stained, respectively. Fig. 1 shows representative cases of high MTH1 expression (MTH1 high, Fig. 1A) and low MTH1 expression (MTH1 low, Fig. 1B).

\subsection{Serum oxidative stress and anti-oxidant capacity (D-ROMs and BAP tests)}

To measure serum oxidative stress (S-OS) and serum anti-oxidant capacity (S-AOC), the total levels of hydroperoxides (R-OOH) and ferric
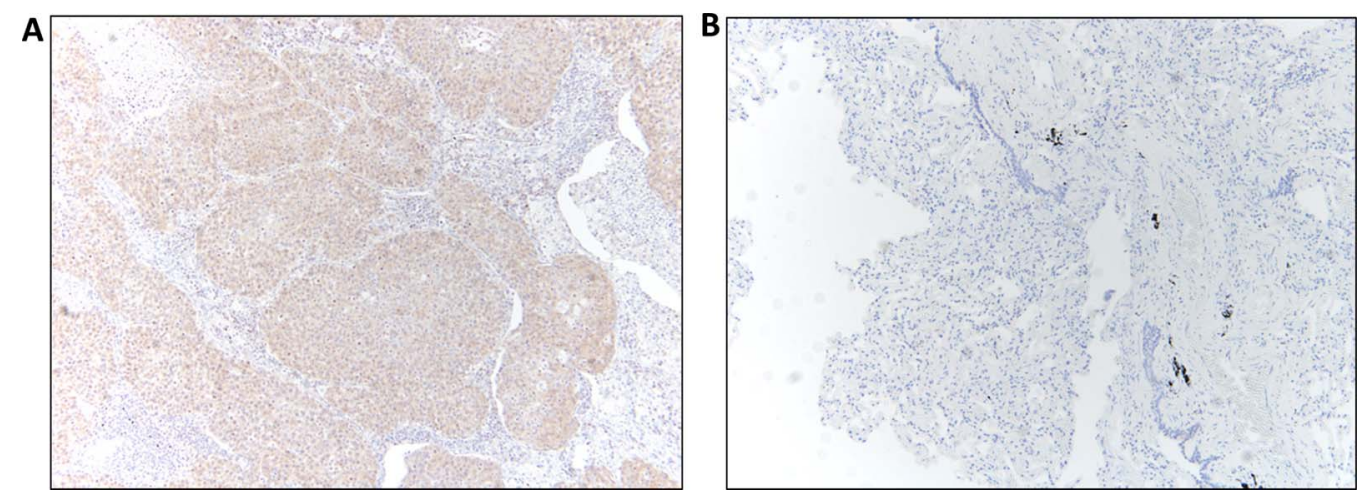

Fig. 1. Immunohistochemical images of typical cases with high (A) and low MTH1 expression (B) (magnification $\times 100)$. 
reducing ability were examined by d-ROM and BAP tests, respectively, which were conducted as reported previously [25].

The d-ROMs test is used to measure the levels of R-OOH, the metabolic products of free radicals and reactive oxygen. In brief, $20 \mu 1$ serum were added to an acetate-buffered solution ( $\mathrm{pH} 4.8)$. The $\mathrm{R}-\mathrm{OOH}$ groups react with the ferric ions $\mathrm{Fe}^{2+}$ and $\mathrm{Fe}^{3+}$ liberated from proteins in the acidic medium and are converted to alkoxy $\left(\mathrm{R}^{-} \mathrm{O}^{-}\right)$and peroxy radicals $\left(\mathrm{R}^{-} \mathrm{OO}^{-}\right)$. These newly formed radicals are trapped chemically by a chromogen ( $N, N$-diethyl-para-phenylendiamine), leading to formation of the corresponding radical cations. The concentration of chromogen can be determined by spectrophotometry (detecting absorption at $505 \mathrm{~nm}$ ). The d-ROMs test was performed using the FRAS 4 analyzer (Health \& Diagnostics Limited Co., Parma, Italy). The reagents for the d-ROMs test were purchased from Wismerll Co., Ltd (Tokyo, Japan).

For the BAP test, in brief, ferric chloride was mixed with a chromogen substrate and a thiocyanate derivative. Serum $(10 \mu \mathrm{l})$ was added to this reaction mixture and incubated at $37^{\circ} \mathrm{C}$ for $5 \mathrm{~min}$. The reduction of ferric ion was quantitated by measuring the change in absorbance at $505 \mathrm{~nm}$, indicating the antioxidant activity of the serum sample. The BAP tests were also performed using the FRAS 4 analyzer. The reagents for the BAP test were purchased from Wismerll Co., Ltd (Tokyo, Japan). The normal value ranges for these tests, as reported by the manufacturer based on non-smokers, are as follows: d-ROMs test, 200-300 U; BAP test: $\geq 2200 \mu \mathrm{mol} / \mathrm{L}$.

\subsection{Statistical analysis}

The associations between MTH1 expression in NSCLC and the patient characteristics were analyzed using Fisher's exact test. Quantitative variables were compared using Student's $t$-tests. OS was defined as the time from the initial surgery to death from any cause, while DFS was defined as the time from the initial surgery to recurrence. The Kaplan-Meier method was used to estimate the survival rates. The resulting Kaplan-Meier curves for each cohort were compared statistically using the log-rank test. Differences with $p$-values $<$ 0.05 were considered significant. Univariate and multivariate analyses of OS and PFS were performed using Cox proportional hazards models and a stepwise method, respectively.

\section{Results}

3.1. Associations between tumor MTH1 expression and clinicopathological characteristics in NSCLC patients

The clinicopathological characteristics of the 197 patients in Cohort A are presented in Table 1 . There were 120 (60.9\%) male and 77 (39.1\%) female patients with a median age of 54 (range 38-83) years. One hundred four patients $(52.8 \%)$ had a smoking history of $>20$ packyears. The majority of the patients had adenocarcinomas $(71.1 \%)$, while $21.3 \%$ and $7.6 \%$ had squamous cell carcinoma (Sq) and other types of NSCLC, respectively. The EGFR mutation status was assessed in 74 tumors; 24 patients (32.4\%) harbored EGFR sensitizing mutations.

In total, 111 patients (56.3\%) showed elevated MTH1 expression in Cohort A. Fisher's exact tests revealed that male patients, a smoking habit of $>20$ pack-years, Sq, advanced pathological stage, tumor diameter $>3 \mathrm{~cm}$, lymph node metastasis, positive pleural invasion, positive lymphatic permeation and positive vascular invasion were significantly associated with high MTH1 expression. Additionally, among the 74 tumors assessed for EGFR mutation status, those with wild-type EGFR tended to be associated with high MTH1 expression $(p=0.0660)$.

Forty-five patients in Cohort A underwent FDG-PET/CT before surgery (Supplementary Table 1). An analysis assessing the association between the maximum standardized uptake value (SUVmax) on FDG-PET/CT and MTH1 protein expression showed an elevated
Table 1

Relationship between clinicopathological characteristics and tumor MTH1 expression in patients with non-small cell lung cancer.

\begin{tabular}{|c|c|c|c|c|c|}
\hline Factor & & $\begin{array}{l}\text { Total } \\
(197)\end{array}$ & $\begin{array}{l}\text { MTH1 } \\
\text { low (86) }\end{array}$ & $\begin{array}{l}\text { MTH1 high } \\
\text { (111) }\end{array}$ & $P$ value \\
\hline \multirow[t]{2}{*}{ Sex } & Male & 120 & 45 & 75 & 0.0297 \\
\hline & Female & 77 & 41 & 36 & \\
\hline \multirow[t]{2}{*}{ Age (years) } & $<75$ & 148 & 66 & 82 & 0.6433 \\
\hline & $\geq 75$ & 49 & 20 & 29 & \\
\hline \multirow{2}{*}{$\begin{array}{l}\text { Smoking status } \\
\text { (pack-years) }\end{array}$} & $<20$ & 93 & 55 & 38 & $<0.0001$ \\
\hline & $\geq 20$ & 104 & 31 & 73 & \\
\hline \multirow[t]{3}{*}{ Histology } & Ad & 140 & 73 & 67 & $<0.0064$ \\
\hline & Sq & 42 & 12 & 30 & vs. Sq) \\
\hline & Others & 15 & 1 & 14 & \\
\hline \multirow[t]{2}{*}{ pStage } & I & 118 & 68 & 50 & $<0.0001$ \\
\hline & $\geq$ II & 79 & 18 & 61 & \\
\hline \multirow[t]{2}{*}{ Tumor diameter } & $<30$ & 105 & 57 & 48 & 0.0012 \\
\hline & $\geq 30$ & 92 & 29 & 63 & \\
\hline \multirow[t]{2}{*}{$\mathrm{N}$ factor } & - & 139 & 72 & 67 & 0.0003 \\
\hline & + & 58 & 14 & 44 & \\
\hline \multirow[t]{2}{*}{$\mathrm{pl}$} & - & 121 & 62 & 59 & 0.0063 \\
\hline & + & 76 & 24 & 52 & \\
\hline \multirow[t]{2}{*}{ ly } & - & 165 & 78 & 87 & 0.0171 \\
\hline & + & 32 & 8 & 24 & \\
\hline \multirow[t]{2}{*}{$\mathrm{v}$} & - & 120 & 65 & 55 & 0.0002 \\
\hline & + & 77 & 21 & 56 & \\
\hline \multirow[t]{2}{*}{ EGFR mutation } & - & 50 & 14 & 36 & $(0.0660)$ \\
\hline & + & 24 & 12 & 12 & \\
\hline
\end{tabular}

MTH1, MutT homolog 1; Ad, adenocarcinoma; Sq, squamous cell carcinoma; pl, pleural invasion; ly, lymphatic permeation; v, vascular invasion; EGFR, epidermal growth factor receptor.

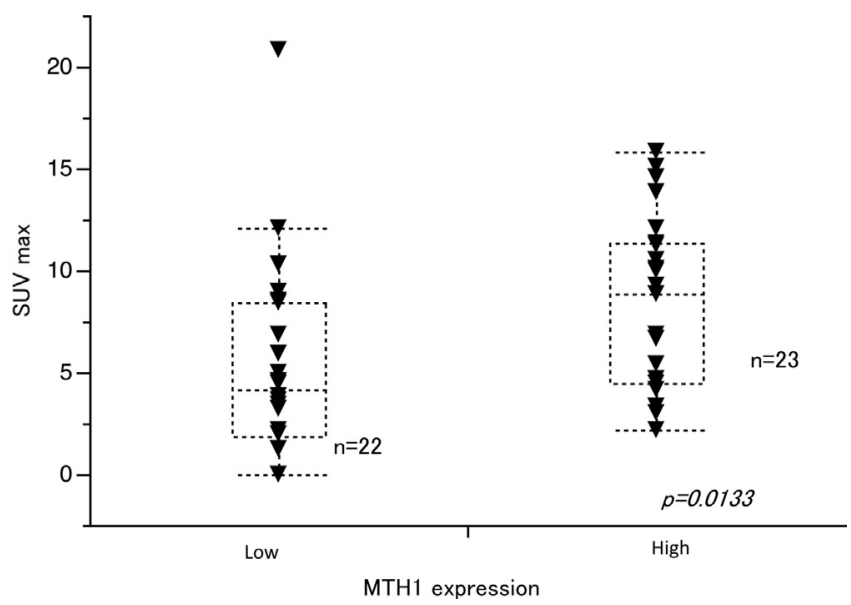

Fig. 2. Relationship between MTH1 expression and SUVmax on FDG-PET.

SUVmax in tumors with high MTH1 expression compared with lowexpressing MTH1 tumors (median SUVmax: $8.9(2.2-15.8)$ and 4.2 (0-20.8), respectively; $p=0.0133$, Fig. 2).

\subsection{Significant difference in OS and DFS according to MTH1 expression in patients with NSCLC}

Survival analysis using Kaplan-Meier curves revealed that patients with high MTH1-expressing NSCLC had a significantly worse prognosis than did those with low MTH1-expressing NSCLC. The 5-year OS rates were $81.6 \%$ and $92.3 \%$, respectively (log-rank test: $p=0.0011$, Fig. 3A) and the 5-year DFS rates $55.0 \%$ and $83.7 \%$, respectively (log-rank test: $p=0.0002$, Fig. 3B).

Specifically, the 5-year OS and DFS rates of patients with high 
(A)

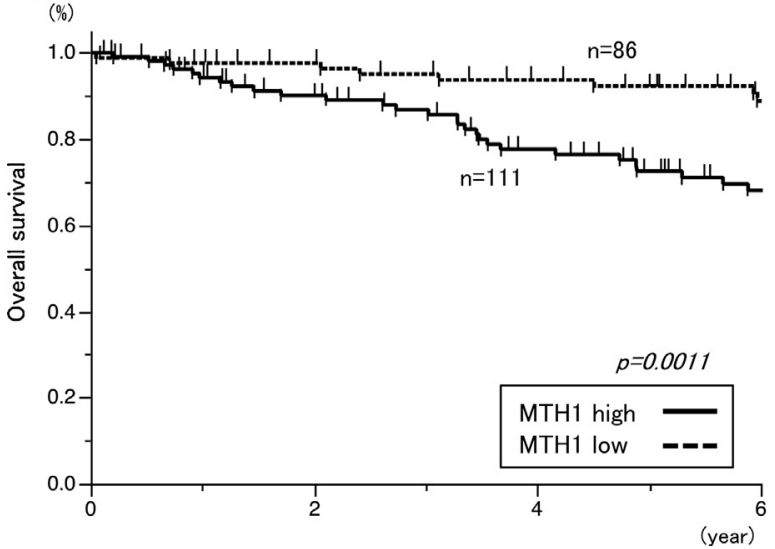

(B)

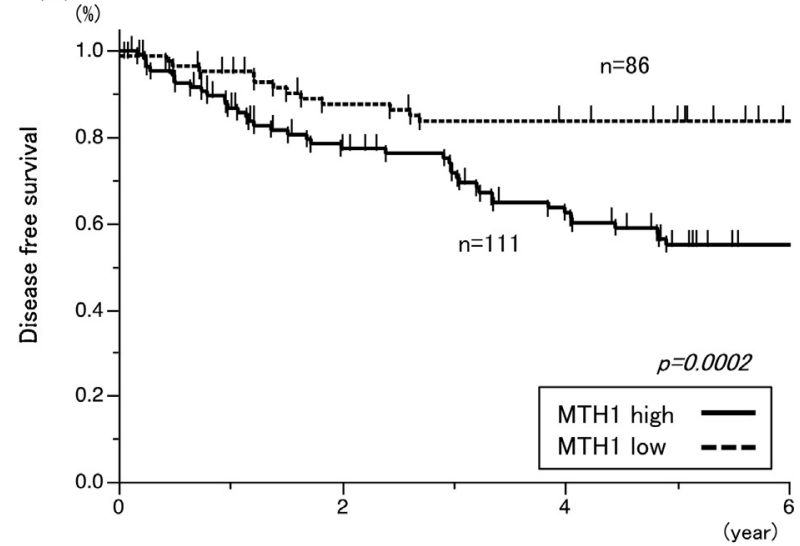

Fig. 3. Kaplan-Meier curves for overall survival (A) and disease-free survival (B) according to the MTH1 expression status in NSCLC.

versus low MTH1-expressing lung adenocarcinoma were $76.5 \%$ versus $95.4 \%$ and $55.3 \%$ versus $85.3 \%$, respectively ( $p=0.0031$ and 0.0007 , respectively; Supplementary Figure 2), whereas MTH1 expression did not significantly affect OS or DFS in patients with a Sq histology (Supplementary Figure 3).

\subsection{Relationships between MTH1 expression in primary tumors and S-OS and S-AOC in NSCLC patients}

In Cohort B, 41 patients underwent the d-ROMs and BAP tests before surgery; 16 and 25 patients had high and low MTH1-expressing tumors, respectively (Supplementary Table 2). The mean values derived from the d-ROMs and BAP tests were 317 (range, 204-514) Carratelli units (U. Carr) and 2308 (1776-3039) $\mu \mathrm{mol} / \mathrm{L}$, respectively. MTH1 high and low patients had median values of 390 (235-514) U. Carr and 303 (219-497) U. Carr in the d-ROMs test, respectively. Median values from the BAP test were 2457 (1964-3039) $\mu \mathrm{mol} / \mathrm{L}$ and 2139 (1659-2768) $\mu \mathrm{mol} / \mathrm{L}$, respectively. Significant differences between MTH1 expression and both oxidative stress and anti-oxidant capacity were observed ( $p=0.0052$ and 0.0015 , respectively; Fig. 4).

\subsection{Univariate and multivariate analysis of predictors of OS and PFS}

On univariate survival analysis, high MTH1-expressing group was associated with an inferior OS and DFS compared with the low MTH1 expressing group (hazard ratio [HR] 3.247, 95\% confidence interval [95\%CI] 1.604-7.721, $p=0.0008$; HR 3.004, 95\%CI 1.683-5.703,

(A) (U. Carr)

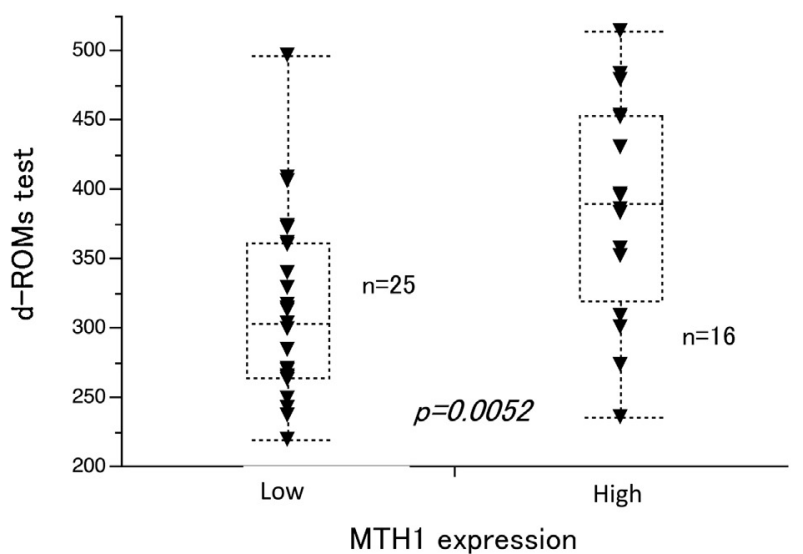

$p=0.0001$, respectively; Supplementary Tables 3 and 4). On multivariate analysis, although patients with high MTH1-expressing tumors tended to have a poor OS and PFS, these associations were not statistically significant (HR 1.713, 95\%CI 0.765-4.118, $p=0.1947$; HR 1.605, 95\%CI 0.827-3.248, $p=0.1639$, respectively).

\section{Discussion}

MTH1 contributes to the maintenance of genetic integrity in somatic cells by sanitizing oxidized nucleotides in the nucleotide pool, preventing cells from incorporating 8-oxoG into DNA during the replication process $[16,28]$. Recent studies have demonstrated that cancer cells are exposed to greater oxidative stress levels than are the surrounding normal cells, and that MTH1 is overexpressed in cancer cells, suggesting that MTH1 contributes to cancer cell proliferation [17-20]. Recently, MTH1 inhibition in cancer cell lines demonstrated that MTH1 activity is required for cancer cell integrity, by preventing incorporation of oxidized dNTPs into DNA [22]. In lung cancer, Kennedy et al. reported that human MTH1 mRNA is overexpressed and inversely correlated with cellular 8-oxo-dG levels [18]. Another study showed that functional MTH1 activity was higher in NSCLC than normal lung tissue [29]. However, the clinical significance of elevated MTH1 protein in NSCLC is not well known. In the present study, we showed that overexpression of the MTH1 protein is associated with the tumor malignant potential, including nodal metastases and advanced stage, in NSCLC patients. These findings are in line with a previous clinical report that evaluated MTH1 mRNA expression in renal cell carcinoma

(B)

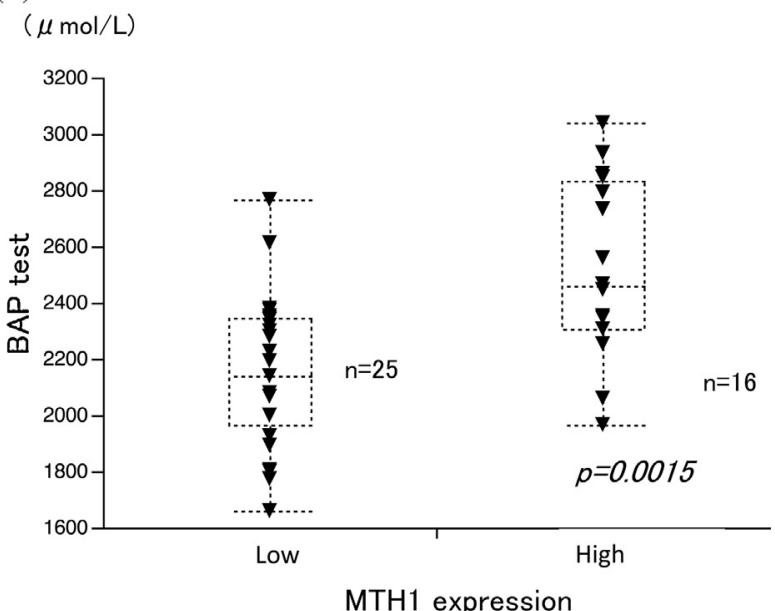

Fig. 4. Relationship between MTH1 expression and oxidative stress (score on the d-ROMs test) (A) and anti-oxidant capacity (score on the BAP test) (B). 
[17]. We also showed that patients with high MTH1 expression had significantly poorer survival than that of patients with low MTH1 expression in terms of both DFS and OS. To our best knowledge, this is the first study to demonstrate the association between MTH1 overexpression and malignant NSCLC traits in a relatively large number of patients. It is not clear why the multivariate analysis failed to show a significant relationship between MTH1 expression and DFS or OS. Since lymph node metastasis ( $\mathrm{N}$ factor) remained an independent predictor for prognosis in the multivariate analysis, we performed a multivariate survival analysis in N0 patients only; however, no statistical significance was detected for the relationship between N factor and MTH1 expression status, possibly because of the lack of statistical power.

A few studies have demonstrated how MTH1 serves as a pro-growth factor in lung cancer [23,30]. Patel et al. reported that RAS-driven NSCLC cells require MTH1 to mitigate the cellular damage caused by ROS. In that study, two findings suggesting the contribution of MTH1 to malignant traits were as follows: 1) in NSCLC harboring functional p53, MTH1 suppression induced oncogenic KRAS-induced genomic DNA strand breaks and inhibited cell proliferation; 2) in NSCLC harboring non-functional p53, MTH1 suppression led to down-regulated KRAS and ROS levels, which reduced Akt signaling for proliferation [23]. Although the KRAS status of the NSCLC patients was not assessed in the present study since the KRAS mutation rate is lower in Asian patients $(<10 \%)$ compared with Caucasian patients $(>25 \%)$ [31], MTH1 expression was significantly increased in the tumors of smokers and was not associated with EGFR mutations. Considering that KRAS mutations are associated with lung cancers in smokers, our data may support a crucial role of MTH1 in KRAS-driven lung cancer.

Elevated expression of MTH1 in tumors with an aggressive phenotype suggests that aggressive cancer cells undergo more oxidative stress than do indolent cancer cells. Studies on cancer metabolism have shown that progressive cancer cells depend largely on aerobic glycolysis (Warburg effect) to achieve rapid replication of DNA and the cellular apparatus, dividing into daughter cells and disseminating to other sites in the body [32]. This metabolic shift is considered to produce more oxidative stress in cancer cells [15]. High FDG intake on FDG-PET generally represents a high metabolic status of the tumor [33]. We showed that high MTH1-expressing NSCLC had a significantly higher SUVmax than that of low MTH1-expressing NSCLC (Fig. 2). Although the number of patients who had evaluable FDF-PET results was small, this is the first study to directly show a positive association between tumor glucose intake and MTH1 expression. Recent FDG-PET studies in lung cancer showed that the SUVmax accurately reflects tumor malignancy [34]. Considering that more aggressive cancers utilize greater amounts of glucose, our findings support that high MTH1 function is required to avoid the oxidative stress produced in hypermetabolic tumors undergoing rapid cell proliferation. Therefore, it is reasonable to consider MTH1 expression as a prognostic marker in not only NSCLC but also other types of cancer.

Our data showed that MTH1 is significantly related to heavy smoking (i.e., $>20$ pack-years), a crucial trigger of oxidative stress. We further observed that the amount of oxidative stress and antioxidant capacity of patients, assessed by serum d-ROMs and BAP tests, respectively, were positively associated with high MTH1 expression in the corresponding lung tumors. Although a previous study from our institution did not find any correlation between smoking status and serum d-ROM test values [25], Asami et al. demonstrated that 8hydroxydeoxyguanosine levels in lung tissues increase according to the amount of smoking [35]. Together with these findings, our present data suggest that lung cancers in smokers have a greater ability to cope with the excessive oxidative stress produced by tobacco smoke. Cancer arising from high oxidative stress environments may have increased MTH1 expression and consequently a great ability to proliferate. Therefore, MTH1 may be a marker of oxidative stress as well as of poor survival in patients with lung cancer $[18,36]$.

In the management of lung cancer, surgery, radiotherapy, and chemotherapy are considered the standard-of-care. Recently, immune checkpoint inhibitors, such as PD-1/PD-L1 antibodies, have become novel and promising therapies for patients with NSCLC [37,38]; however, new therapeutic strategies are needed to prolong the prognosis of NSCLC patients. Patel and colleagues reported that blockade of MTH1 contributed to the suppression of cell proliferation and epithelial-mesenchymal transition (EMT), supporting MTH1 as a therapeutic target in patients with NSCLC [23]. Given that $41 \%$ of patients were positive for MTH1 expression in this study, some populations with NSCLC might be effective responders to MTH1 inhibition. Huber et al. reported that (s)-crizotinib inhibited MTH1 and suppressed tumor growth in vivo $[24,39]$. The potential of MTH1 as a therapeutic target should be elucidated in further studies.

Our study has several limitations: first, this was a retrospective study conducted in a single institution; second, even though methodological issues with immunohistochemistry and the evaluation criteria for MTH1 were examined, they should be further validated in further studies. Establishment of reliable criteria for MTH1 expression status will help to distinguish clinically significant subgroups.

\section{Conclusions}

In conclusion, we showed more malignant traits and a worse prognosis in high than in low MTH1-expressing NSCLC. MTH1 positivity was associated with tumors in smokers and oxidative stress levels in patients. The MTH1 expression status may help identify patients with poorer prognoses and those who will benefit from MTH1 targeted therapy.

\section{Appendix A. Supplementary data}

Supplementary data associated with this article can be found, in the online version, at http://dx.doi.org/10.1016/j.lungcan.2017.04.012.

\section{References}

[1] L.A. Torre, F. Bray, R.L. Siegel, J. Ferlay, J. Lortet-Tieulent, A. Jemal, Global cancer statistics, 2012, CA Cancer J. Clin. 65 (2015) 87-108.

[2] A.E. Karnoub, R.A. Weinberg, Ras oncogenes: split personalities, Nat. Rev. Mol. Cell Biol. 9 (2008) 517-531.

[3] M. Soda, Y.L. Choi, M. Enomoto, S. Takada, Y. Yamashita, S. Ishikawa, et al., Identification of the transforming EML4-ALK fusion gene in non-small-cell lung cancer, Nature 448 (2007) 561-566.

[4] J.G. Paez, P.A. Janne, J.C. Lee, S. Tracy, H. Greulich, S. Gabriel, et al., EGFR mutations in lung cancer: correlation with clinical response to gefitinib therapy, Science 304 (2004) 1497-1500.

[5] E.L. Kwak, Y.J. Bang, D.R. Camidge, A.T. Shaw, B. Solomon, R.G. Maki, et al., Anaplastic lymphoma kinase inhibition in non-small-cell lung cancer, N. Engl. J. Med. 363 (2010) 1693-1703.

[6] T.J. Lynch, D.W. Bell, R. Sordella, S. Gurubhagavatula, R.A. Okimoto, B.W. Brannigan, et al., Activating mutations in the epidermal growth factor receptor underlying responsiveness of non-small-cell lung cancer to gefitinib, N. Engl. J. Med. 350 (2004) 2129-2139.

[7] X. Lu, Y. Kang, Hypoxia and hypoxia-inducible factors: master regulators of metastasis, Clin. Cancer Res. 16 (2010) 5928-5935.

[8] D. Ziech, R. Franco, A. Pappa, Panayiotidis MI, Reactive oxygen species (ROS)induced genetic and epigenetic alterations in human carcinogenesis, Mutat. Res. 711 (2011) 167-173.

[9] B.N. Ames, M.K. Shigenaga, T.M. Hagen, Oxidants, antioxidants, and the degenerative diseases of aging, Proc. Natl. Acad. Sci. U. S. A. 90 (1993) 7915-7922.

[10] Y. Nakabeppu, K. Sakumi, K. Sakamoto, D. Tsuchimoto, T. Tsuzuki, Y. Nakatsu, Mutagenesis and carcinogenesis caused by the oxidation of nucleic acids, Biol. Chem. 387 (2006) 373-379.

[11] Y. Nakabeppu, Cellular levels of 8-oxoguanine in either DNA or the nucleotide pool play pivotal roles in carcinogenesis and survival of cancer cells, Int. J. Mol. Sci. 15 (2014) 12543-12557.

[12] Y. Nakabeppu, Molecular genetics and structural biology of human MutT homolog, MTH1, Mutat. Res. 477 (2001) 59-70.

[13] K. Sakumi, M. Furuichi, T. Tsuzuki, T. Kakuma, S. Kawabata, H. Maki, et al., Cloning and expression of cDNA for a human enzyme that hydrolyzes 8-oxo-dGTP, a mutagenic substrate for DNA synthesis, J. Biol. Chem. 268 (1993) 23524-23530.

[14] T. Tsuzuki, A. Egashira, H. Igarashi, T. Iwakuma, Y. Nakatsuru, Y. Tominaga, et al., Spontaneous tumorigenesis in mice defective in the MTH1 gene encoding 8-oxodGTPase, Proc. Natl. Acad. Sci. U. S. A. 98 (2001) 11456-11461.

[15] R.A. Cairns, I.S. Harris, T.W. Mak, Regulation of cancer cell metabolism, Nat. Rev. 
Cancer 11 (2011) 85-95.

[16] S. Oka, M. Ohno, D. Tsuchimoto, K. Sakumi, M. Furuichi, Y. Nakabeppu, Two distinct pathways of cell death triggered by oxidative damage to nuclear and mitochondrial DNAs, EMBO J. 27 (2008) 421-432.

[17] K. Okamoto, S. Toyokuni, W.J. Kim, O. Ogawa, Y. Kakehi, S. Arao, et al., Overexpression of human mutT homologue gene messenger RNA in renal-cell carcinoma: evidence of persistent oxidative stress in cancer, Int. J. Cancer 65 (1996) $437-441$.

[18] C.H. Kennedy, R. Cueto, S.A. Belinsky, J.F. Lechner, W.A. Pryor, Overexpression of hMTH1 mRNA: a molecular marker of oxidative stress in lung cancer cells, FEBS Lett. 429 (1998) 17-20.

[19] T. Iida, A. Furuta, M. Kawashima, J. Nishida, Y. Nakabeppu, T. Iwaki, Accumulation of 8-oxo-2'-deoxyguanosine and increased expression of hMTH1 protein in brain tumors, Neuro Oncol. 3 (2001) 73-81.

[20] W.J. Song, P. Jiang, J.P. Cai, Z.Q. Zheng, Expression of Cytoplasmic 8-oxo-Gsn and MTH1 Correlates with Pathological Grading in Human Gastric Cancer, Asian Pac. J. Cancer Prev. 16 (2015) 6335-6338.

[21] E. Coskun, P. Jaruga, A.S. Jemth, O. Loseva, L.D. Scanlan, A. Tona, et al., Addiction to MTH1 protein results in intense expression in human breast cancer tissue as measured by liquid chromatography-isotope-dilution tandem mass spectrometry, DNA Repair (Amst) 33 (2015) 101-110.

[22] H. Gad, T. Koolmeister, A.S. Jemth, S. Eshtad, S.A. Jacques, C.E. Strom, et al., MTH1 inhibition eradicates cancer by preventing sanitation of the dNTP pool, Nature 508 (2014) 215-221.

[23] A. Patel, D.G. Burton, K. Halvorsen, W. Balkan, T. Reiner, C. Perez-Stable, et al., MutT Homolog 1 (MTH1) maintains multiple KRAS-driven pro-malignant pathways, Oncogene 34 (2015) 2586-2596.

[24] K.V. Huber, E. Salah, B. Radic, M. Gridling, J.M. Elkins, A. Stukalov, et al., Stereospecific targeting of MTH1 by (S)-crizotinib as an anticancer strategy, Nature 508 (2014) 222-227.

[25] K. Ito, T. Yano, Y. Morodomi, T. Yoshida, M. Kohno, A. Haro, et al., Serum antioxidant capacity and oxidative injury to pulmonary DNA in never-smokers with primary lung cancer, Anticancer Res. 32 (2012) 1063-1067.

[26] D. Kang, J. Nishida, A. Iyama, Y. Nakabeppu, M. Furuichi, T. Fujiwara, et al., Intracellular localization of 8-oxo-dGTPase in human cells, with special reference to the role of the enzyme in mitochondria, J. Biol. Chem. 270 (1995) 14659-14665.

[27] S. Akiyama, H. Saeki, Y. Nakashima, M. Iimori, H. Kitao, E. Oki, et al., Prognostic impact of MutT homolog-1 expression on esophageal squamous cell carcinoma, Cancer Med. 6 (2017) 258-266.

[28] J. Ichikawa, D. Tsuchimoto, S. Oka, M. Ohno, M. Furuichi, K. Sakumi, et al., Oxidation of mitochondrial deoxynucleotide pools by exposure to sodium nitro- prusside induces cell death, DNA Repair (Amst) 7 (2008) 418-430.

[29] E. Speina, K.D. Arczewska, D. Gackowski, M. Zielinska, A. Siomek, J. Kowalewski, et al., Contribution of hMTH1 to the maintenance of 8-oxoguanine levels in lung DNA of non-small-cell lung cancer patients, J. Natl. Cancer Inst. 97 (2005) 384-395.

[30] M.G. Giribaldi, A. Munoz, K. Halvorsen, A. Patel, P. Rai, MTH1 expression is required for effective transformation by oncogenic HRAS, Oncotarget 6 (2015) 11519-11529.

[31] L.M. Sholl, D.L. Aisner, M. Varella-Garcia, L.D. Berry, D. Dias-Santagata, I.I. Wistuba, et al., Multi-institutional oncogenic driver mutation analysis in lung adenocarcinoma: the lung cancer mutation consortium experience, J. Thorac Oncol. 10 (2015) 768-777.

[32] M.G. Vander Heiden, L.C. Cantley, C.B. Thompson, Understanding the Warburg effect: the metabolic requirements of cell proliferation, Science 324 (2009) 1029-1033.

[33] F. Fathinul, A.J. Nordin, W.F. Lau, 18[F]FDG-PET/CT is a useful molecular marker in evaluating tumour aggressiveness: a revised understanding of an in-vivo FDGPET imaging that alludes the alteration of cancer biology, Cell Biochem. Biophys. 66 (2013) 37-43.

[34] T. Berghmans, M. Dusart, M. Paesmans, C. Hossein-Foucher, I. Buvat, C. Castaigne, et al., Primary tumor standardized uptake value (SUVmax) measured on fluorodeoxyglucose positron emission tomography (FDG-PET) is of prognostic value for survival in non-small cell lung cancer (NSCLC): a systematic review and metaanalysis (MA) by the European Lung Cancer Working Party for the IASLC Lung Cancer Staging Project, J. Thorac Oncol. 3 (2008) 6-12.

[35] S. Asami, H. Manabe, J. Miyake, Y. Tsurudome, T. Hirano, R. Yamaguchi, et al., Cigarette smoking induces an increase in oxidative DNA damage, 8-hydroxydeoxyguanosine, in a central site of the human lung, Carcinogenesis 18 (1997) 1763-1766.

[36] S. Toyokuni, Novel aspects of oxidative stress-associated carcinogenesis, Antioxid Redox Signal. 8 (2006) 1373-1377.

[37] R.S. Herbst, P. Baas, D.W. Kim, E. Felip, J.L. Perez-Gracia, J.Y. Han, et al., Pembrolizumab versus docetaxel for previously treated PD-L1-positive, advanced non-small-cell lung cancer (KEYNOTE-010): a randomised controlled trial, Lancet (2015).

[38] J. Brahmer, K.L. Reckamp, P. Baas, L. Crino, W.E. Eberhardt, E. Poddubskaya, et al., Nivolumab versus Docetaxel in Advanced Squamous-Cell Non-Small-Cell Lung Cancer, N. Engl. J. Med. 373 (2015) 123-135.

[39] Y. Niu, D. Pan, D. Shi, Q. Bai, H. Liu, X. Yao, Influence of chirality of crizotinib on its MTH1 protein inhibitory activity: insight from molecular dynamics simulations and binding free energy calculations, PLoS ONE 10 (2015) 0145219. 